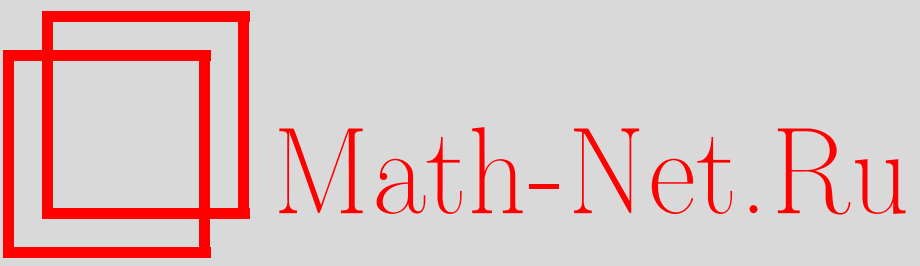

Э. Ю. Лернер, М. Д. Миссаров, Глобальный поток ренормализационной группы и термодинамический предел в фермионной иерархической модели, ТМФ, 1996 , том 107, номер 2, 201-212

DOI: https://doi.org/10.4213/tmf1149

Использование Общероссийского математического портала Math-Net.Ru подразумевает, что вы прочитали и согласны с пользовательским соглашением

http://www . mathnet.ru/rus/agreement

Параметры загрузки:

IP: 54.198 .55 .26

26 апреля 2023 г., 09:29:59 


\title{
ГЛОБАЛЬНЫЙ ПОТОК РЕНОРМАЛИЗАЦИОННОЙ ГРУППЫ И ТЕРМОДИНАМИЧЕСКИЙ ПРЕДЕЛ В ФЕРМИОННОЙ ИЕРАРХИЧЕСКОЙ МОДЕЛИ
}

\begin{abstract}
Преобразование ренормализационной группы в фермионной иерархической модели задается рациональным отображением в плоскости констант связи. Мы исследуем глобальную динамику этого отображения. Доказано существование термодинамического предела в исследуемых областях констант связи. Изучен вопрос об автомодельном пределе построенных полей.
\end{abstract}

\section{1. ВВЕДЕНИЕ}

В данной работе мы продолжаем исследование свойств фермионной иерархической $(\bar{\psi} \psi)^{2}$-модели Дайсона, введенной в статье [1] (обобщение этой модели на случай произвольного числа компонент поля см. в [2]). В частности, там было показано, что преобразование ренормализационной группы (РГ) описывается как рациональное преобразование в пространстве констант связи. Особенно простой вид этого преобразования в четырехкомпонентной $(\bar{\psi} \psi)^{2}$-модели позволил явно описать все ветви неподвижных точек РГ. В некотором смысле эта модель является точно решаемой и допускает исследование термодинамических свойств для произвольных значений констант связи. Она сочетает все преимушества иерархических моделей для [3-5] с комбинаторными свойствами грассманово-значных спиновых моделей типа Гросса-Неве [6, 7]. Отметим, что вырожденный случай иерархической фермионной модели при некотором выделенном значении параметра РГ был ранее изучен Дорласом [8].

Напомним основные определения. Пусть $\mathbb{N}$ - множество натуральных чисел, $V_{k, s}=$ $\left\{j: \quad j \in \mathbb{N},(k-1) n^{s}<j \leq k n^{s}\right\}, k \in \mathbb{N}, s \in \mathbb{N}$, и пусть $s(i, j)=\min \{s:$ сушествует $k$ такое, что $\left.i \in V_{k, s}, j \in V_{k, s}\right\}$. Иерархическое расстояние $d(i, j), i, j \in \mathbb{N}$, определяется по формуле $d(i, j)=n^{s(i, j)}$, если $i \neq j, d(i, i)=0$. Рассмотрим четырехкомпонентное фермионное поле $\psi^{*}(i)=\left(\bar{\psi}_{1}(i), \psi_{1}(i), \bar{\psi}_{2}(i), \psi_{2}(i)\right), i \in \mathbb{N}$, где компоненты суть образуюшие грассмановой алгебры. Блок-спиновое РГ-преобразование определяется по формуле

$$
r_{\alpha} \psi^{*}(i)=n^{-\alpha / 2} \sum_{j \in V_{i, 1}} \psi^{*}(j),
$$

где $\alpha$ есть параметр РГ. Мы переобозначим $V_{1, N}$ через $\Lambda_{N}$, и пусть $A_{N}$ будет грассмановой подалгеброй, порожденной $4 n^{N}$ генераторами, соответствуюшими этому подобъему. 
Мы определим гиббсовское состояние ("математическое ожидание") $\rho_{N}(r, g)$ на $A_{N}$ как

$$
\left(\rho_{N}(\alpha ; r, g)\right)(F)=Z_{N}^{-1}(r, g) \int F\left(\psi^{*}\right) \exp \left\{-H_{0, N}\left(\psi^{*} ; \alpha\right)\right\} \prod_{i \in \Lambda_{N}} f\left(\psi^{*}(i) ; r, g\right) d \psi^{*}(i)
$$

где $d \psi^{*}=d \psi_{1} d \bar{\psi}_{1} d \psi_{2} d \bar{\psi}_{2}$, и мы используем обычные правила интегрирования по антикоммутирующим переменным $[9], F \in A_{N}$,

$$
\begin{aligned}
H_{0, N}\left(\psi^{*} ; \alpha\right) & =\sum_{i, j \in \Lambda_{N}} d_{0, N}(i, j)\left(\bar{\psi}_{1}(i) \psi_{1}(j)+\bar{\psi}_{2}(i) \psi_{2}(j)\right), \\
d_{0, N}(i, j) & =d_{0}(i, j)-c(N), \quad c(N)=\frac{\left(1-n^{\alpha-1}\right)^{2}}{\left(1-n^{-\alpha}\right)\left(1-n^{-1}\right)} n^{-\alpha(N+1)}, \\
d_{0}(i, i) & =\frac{1-n^{-1}}{1-n^{-\alpha}}, \quad d_{0}(i, j)=\frac{1-n^{\alpha-1}}{1-n^{-\alpha}} d^{-\alpha}(i, j), \quad i \neq j, \\
f\left(\psi^{*}(i) ; r, g\right) & =\exp \left\{-\left(r\left(\bar{\psi}_{1}(i) \psi_{1}(i)+\bar{\psi}_{2}(i) \psi_{2}(i)\right)+g\left(\bar{\psi}_{1}(i) \psi_{1}(i) \bar{\psi}_{2}(i) \psi_{2}(i)\right)\right)\right\}, \\
Z_{N}(r, g) & =\int \exp \left\{-H_{0, N}\left(\psi^{*} ; \alpha\right)\right\} \prod_{i \in \Lambda_{N}} f\left(\psi^{*}(i) ; r, g\right) d \psi^{*}(i) .
\end{aligned}
$$

Тогда для ренормированного состояния $\rho^{\prime}(F)=\rho\left(F\left(r_{\alpha} \psi^{*}\right)\right), F \in A_{N-1}$, можно показать (см. [1]), что

$$
\rho_{N}^{\prime}\left(H_{N}\left(\psi^{*} ; \alpha ; r, g\right)\right)=\rho_{N-1}\left(H_{N-1}\left(\psi^{*} ; \alpha ; r^{\prime}, g^{\prime}\right)\right)
$$

где

$$
r^{\prime}=n^{\alpha-1}\left(\frac{(r+1)^{2}-g}{(r+1)^{2}-g / n}(r+1)-1\right), \quad g^{\prime}=n^{2 \alpha-3}\left(\frac{(r+1)^{2}-g}{(r+1)^{2}-g / n}\right)^{2} g .
$$

РГ-преобразование (1) имеет две ветви нетривиальных неподвижных точек $\left(r_{ \pm}(\alpha)\right.$, $\left.g_{ \pm}\left(r_{ \pm}(\alpha)\right)\right)$, где

$$
r_{ \pm}(\alpha)=\frac{ \pm \sqrt{n}-n^{\alpha-1}}{1 \mp \sqrt{n}}, \quad g_{ \pm}(r)=\frac{r(1+r)^{2}}{1+r \pm 1 / \sqrt{n}}, \quad \alpha \neq 1 .
$$

При $\alpha=1$ эти ветви проходят через точку $(-1,0)$, которую мы исключаем из нашего рассмотрения ввиду того, что $Z_{N}(-1,0)=0$. При $\alpha=3 / 2$ "плюсовая" ветвь неподвижных точек пересекается с тривиальной ( “гауссовой”) ветвью неподвижных точек $r \equiv 0$, $g \equiv 0$. При $\alpha$, стремящемся к $1 / 2$, значения $g_{+}$стремятся к плюс или минус бесконечности, но, уточняя [1], мы можем сказать, что при $\alpha=1 / 2$ "плюсовая" ветвь проходит через состояние, задаваемое $\delta$-функцией, которой соответствует грассманово-значная "плотность"

$$
\delta\left(\psi^{*}(i)\right)=\bar{\psi}_{1}(i) \psi_{1}(i) \bar{\psi}_{2}(i) \psi_{2}(i) .
$$

Заметим, что это состояние является вырожденным в том смысле, что оно отлично от нуля только на подпространстве констант.

Верхняя полуплоскость $g>0$ разбивается на 3 части: 
часть, лежащую слева от устойчивой инвариантной кривой для “минусовой” неподвижной точки $\left(r_{-}, g_{-}\right)$;

часть, лежашую справа от устойчивой инвариантной кривой для точки $\left(r_{+}, g_{+}\right)$(при $\alpha>3 / 2)$ или для точки $(0,0)$ (при $1<\alpha \leq 3 / 2)$;

часть, лежашую между ними.

В этой работе мы опишем глобальную динамику РГ в первых двух частях. Вопрос о поведении РГ в последней части является более сложным и находится в стадии исследования. Мы также исследуем динамику РГ в нижней полуплоскости при $\alpha \geq 3 / 2$. При $\alpha<3 / 2$ "плюсовая" неподвижная точка переходит в нижнюю полуплоскость и является при некоторых значениях $\alpha$ неустойчивым фокусом, и мы также не будем обсуждать этот случай в данной статье.

Эти результаты позволяют доказать существование термодинамического предела в рассматриваемых областях. Кроме того, из анализа динамики РГ следует, что все эти области являются зоной притяжения состояния, задаваемого $\delta$-функцией. Если использовать аналогию с теорией случайных полей, можно сказать, что автомодельный предел фермионных полей является вырожденным полем с независимыми значениями.

\section{2. ГЛОБАЛЬНЫЙ ПОТОК РГ}

Введем следующие обозначения. Пусть

$$
\begin{aligned}
g_{1}^{ \pm}(r) & =\frac{(r+1)(n \mp \sqrt{n})-n+n^{2-\alpha}}{(r+1)(n \mp \sqrt{n})-1+n^{1-\alpha}}(r+1)^{2}, \\
g_{2}(r) & =\frac{\left(1-n^{1-\alpha}\right) r}{\left(1-n^{-\alpha}\right) r+1-n^{-1}}(r+1)^{2}, \\
g_{3}^{ \pm}(r) & =\frac{n^{\alpha-1 / 2} \mp n}{n^{\alpha-1 / 2} \mp 1}(r+1)^{2}, \\
g_{4}(r) & =\frac{r\left(1-n^{\alpha-2}\right)}{r\left(1-n^{\alpha-2}\right)+1-n^{-1}}(r+1)^{2}
\end{aligned}
$$

(см. рисунок). Прямым вычислением легко проверить справедливость следующих формул:

$$
\begin{gathered}
\frac{\left(r^{\prime}(r, g)+1\right)^{2}}{g^{\prime}(r, g)}-\frac{(r+1)^{2}}{g}=\frac{n-1}{g\left(g-(r+1)^{2}\right)^{2}}\left(r+1-\frac{1-n^{1-\alpha}}{n-\sqrt{n}}\right) \times \\
\times\left(r+1-\frac{1-n^{1-\alpha}}{n+\sqrt{n}}\right)\left(g-g_{1}^{+}(r)\right)\left(g-g_{1}^{-}(r)\right) \\
r^{\prime}(r, g)-r=n^{\alpha}\left(\left(1-n^{-\alpha}\right) r+1-n^{-1}\right)\left(g-g_{2}(r)\right), \\
g^{\prime}(r, g)-g=\frac{g\left(n^{2 \alpha-1}-1\right)}{g-n(r+1)^{2}}\left(g-g_{3}^{+}(r)\right)\left(g-g_{3}^{-}(r)\right) .
\end{gathered}
$$

Из формулы (2) следует, что кривые $g_{1}^{ \pm}(r)$ характеризуются тем, что одна итерация отображения (1) сохраняет отношение $(r+1)^{2} / g$; знак плюс (минус) означает, что соответствуюшая кривая проходит через “плюсовую" ("минусовую”) неподвижную точку. Аналогично из формулы (3) (формулы (4)) следует, что кривая $g_{2}(r)$ (кривые $g_{1}^{ \pm}(r)$ и 


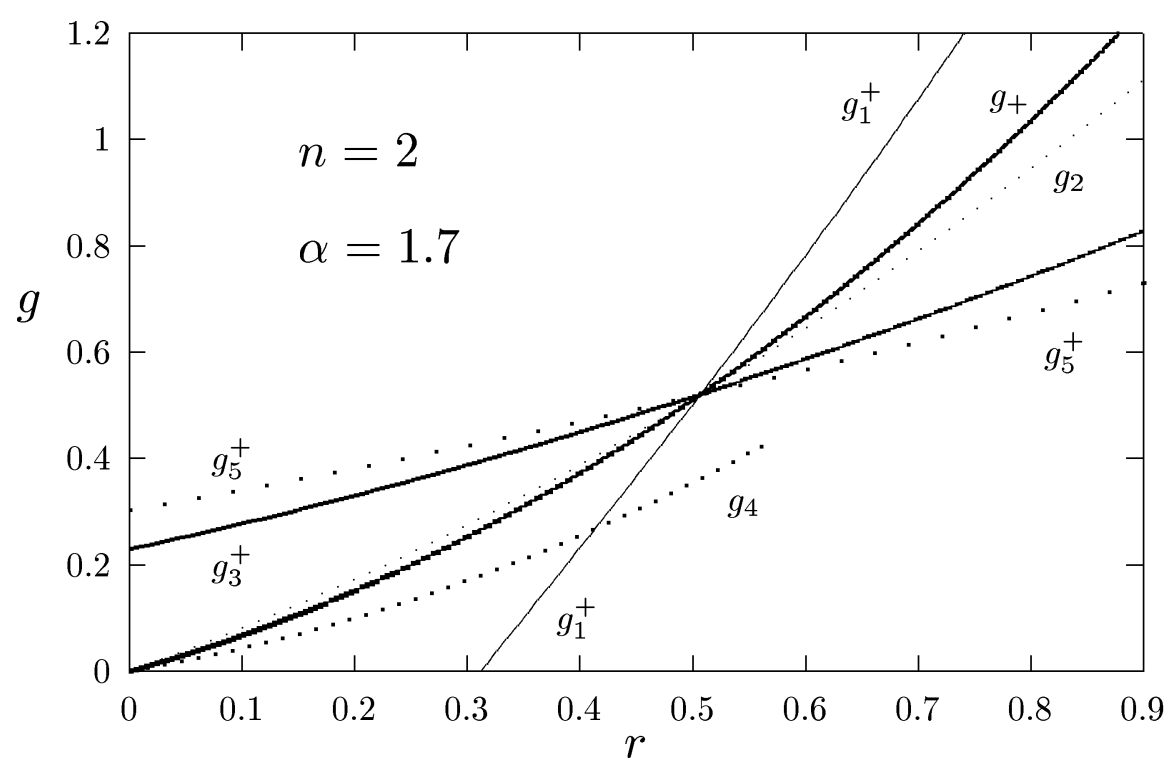

Взаимное расположение рассматриваемых кривых в первом квадранте при $\alpha>3 / 2$

ось $r)$ есть множество точек $(r, g)$, для которых одна итерация отображения $(1)$ сохраняет значение $r$ (соответственно значение $g$ ).

Можно показать (см. [1]), что в “плюсовой” неподвижной точке при $\alpha>3 / 2$ старшее собственное число дифференциала РГ больше единицы, а младшее положительно и меньше единицы. В “минусовой” неподвижной точке при $\alpha>1$ старшее собственное число дифференциала РГ больше единицы, а младшее отрицательно и больше чем -1 . Собственные числа дифференциала РГ в тривиальной неподвижной точке равны $n^{\alpha-1}$ и $n^{2 \alpha-3}$. Из общей теории динамических систем (см. [10]) следует локальное существование и единственность устойчивых инвариантных кривых, проходящих через точку $\left(r_{+}, g_{+}\right)$при $\alpha>3 / 2$, через тривиальную неподвижную точку при $1<\alpha<3 / 2$ и через точку $\left(r_{-}, g_{-}\right)$при $\alpha>1$. Оказывается, что в нашем случае можно описать глобальное поведение этих инвариантных кривых.

Пусть

$$
\begin{aligned}
& G_{1}=\left\{(r, g): \quad 0 \leq r \leq r_{+}(\alpha), \quad \max \left\{0, g_{4}(r), g_{1}^{+}(r)\right\} \leq g \leq g_{+}(r)\right\} \cup \\
& \cup\left\{(r, g): \quad \max \left\{0, r_{+}(\alpha)\right\} \leq r, \quad g_{+}(r) \leq g \leq g_{1}^{+}(r)\right\}, \\
& G_{2}=\left\{(r, g): \quad r \leq r_{-}(\alpha), \quad g_{1}^{-}(r) \leq g \leq g_{-}(r)\right\} \cup \\
& \cup\left\{(r, g): \quad r_{-}(\alpha)<r<-1, \quad g_{-}(r)<g<g_{1}^{-}(r)\right\}, \\
& G_{3}=\left\{(r, g): \quad-\frac{1-n^{-1}}{1-n^{-\alpha}}<r \leq 0, \quad g_{2}(r) \leq g \leq 0\right\} .
\end{aligned}
$$

ТЕОрема 1. Существует гладкая возрастающая функиия $g=h_{+}(r ; \alpha)$, $0 \leq r<\infty$, график которой лежит в области $G_{1}$ и является частью $\gamma_{1}$ устойчивой РГ-инвариантной кривой для "плюсовой" неподвижной точки при $\alpha>3 / 2$ и для тривиальной неподвижной точки при $3 / 2 \geq \alpha>1$. Существует гладкая убъвающая функция $g=h_{-}(r ; \alpha),-\infty<r<-1$, график которой лежст в области $G_{2}$ 
и является частью $\gamma_{2}$ устойчивой $Р Г$-инвариантной кривой для “минусовой” неподвижной точки при $\alpha>1$. При $\alpha \geq 3 / 2$ для неподвижной точки $(0,0)$ существует единственная неустойчивая РГ-инвариантная кривая $\gamma_{3}$, принадлежащая области $G_{3}$. Эта кривая уходит по $g$ на $-\infty$ и делит нижнюю полуплоскость на две связные части. Кроме того, все точки из множсеств $G_{i} \backslash \gamma_{i}$ за конечное число итераций РГ выходят из областей $G_{i}, \quad i=1,2,3$. Образы кривых $\gamma_{1}$ и $\gamma_{2}$ после первой итерации РГ суть множества

$$
\left\{(r, g): \quad(r, g) \in \gamma_{1}, \quad \frac{g}{\left(r+n^{\alpha-1}\right)^{2}}<\frac{1}{n}\right\}
$$

$u$

$$
\left\{(r, g): \quad(r, g) \in \gamma_{2}, \quad \frac{1}{n}<\frac{g}{\left(r+n^{\alpha-1}\right)^{2}}<1\right\},
$$

соответственно. Образ кривой $\gamma_{3}$ совпадает $с \gamma_{3}$.

Утверждения теоремы 1 принадлежат одному из авторов (М.Д.М.), и их доказательства будут опубликованы в отдельной работе.

Заметим, что кривая $\gamma_{1}$ проходит через $(0,0)$; область правее этой кривой обозначим через $\Omega_{1}$

$$
\Omega_{1}=\left\{(r, g): \quad r>0, \quad 0 \leq g<h_{+}(r ; \alpha)\right\} .
$$

Кривая $\gamma_{2}$ входит в $(-1,0)$; область левее этой кривой обозначим через $\Omega_{2}$ :

$$
\Omega_{2}=\left\{(r, g): \quad r<-1, \quad 0 \leq g<h_{-}(r ; \alpha)\right\} .
$$

Часть нижней полуплоскости $g \leq 0$ справа (слева) от кривой $\gamma_{3}$ мы обозначим через $\Omega_{3}$ (через $\Omega_{4}$ ).

Обозначим через $\left(r^{(N)}, g^{(N)}\right)$ образ точки $(r, g)=\left(r^{(0)}, g^{(0)}\right)$ при $N$ итерациях отображения (1). Пусть

$$
\beta^{(N)}=\frac{g^{(N)}}{\left(r^{(N)}+1\right)^{2}}, \quad \sigma^{(N)}=\frac{1-\beta^{(N)}}{1-\beta^{(N)} / n} .
$$

Заметим, что если для некоторой точки $(r, g)$ выполнено $g^{\left(N_{0}\right)}=0$, то

$$
g^{(N)}=0, \quad r^{(N)}=r^{\left(N_{0}\right)} n^{\left(N-N_{0}\right)(\alpha-1)}
$$

для всех $N \geq N_{0}$.

Tеорема 2. Пусть $\alpha>1 u(r, g) \in \Omega_{1} \cup \Omega_{2}$ uлu $\alpha \geq 3 / 2 u(r, g) \in \Omega_{3} \cup \Omega_{4}$. Тогда существуют константы $c_{1}\left(r^{(0)}, g^{(0)}\right)$ и $c_{2}\left(r^{(0)}, g^{(0)}\right)$, такие что

$$
\lim _{N \rightarrow \infty} \frac{r^{(N)}}{n^{N(\alpha-1)}}=c_{1}, \quad \lim _{N \rightarrow \infty} \frac{g^{(N)}}{n^{N(2 \alpha-3)}}=c_{2} .
$$

При әтом $\mathrm{c}_{1}>0$, если $(r, g) \in \Omega_{1} \cup \Omega_{3} ; c_{1}<0$, если $(r, g) \in \Omega_{2} \cup \Omega_{4} ;$ константа $c_{2}$ равняется нулю тогда и только тогда, когда $g^{(N)}=0$ для некоторого $N \geq 0$.

Прежде чем доказывать теорему, докажем несколько вспомогательных утверждений. 
Лемма 1. Области $\Omega_{1}$ и $\Omega_{2}$ при $\alpha>1$ и области $\Omega_{3}, \Omega_{4}$ при $\alpha \geq 3 / 2$ переходят сами в себя при преобразовании РГ.

ДоКАЗАТЕЛЬСТВо.Докажем это утверждение для области $\Omega_{2}$ (доказательство для других областей проводится аналогично). Заметим сначала, что если

$$
(r, g) \in \Omega_{2}^{\prime} \equiv\left\{(r, g): \quad r<-1, \quad 0<g<(r+1)^{2}\right\},
$$

то $\left(r^{\prime}, g^{\prime}\right) \in \Omega_{2}^{\prime}$, так как

$$
\begin{aligned}
r^{\prime}+1 & <n^{\alpha-1} \sigma(r+1), \\
g^{\prime} & =n^{2 \alpha-3} \sigma^{2} g .
\end{aligned}
$$

Докажем теперь, что образ области

$$
\Omega_{2}^{\prime \prime} \equiv\left\{(r, g): \quad(r, g) \in \Omega_{2}, \quad g>(r+1)^{2}\right\}
$$

содержится в $\Omega_{2}$. Допустим, что это не так, т.е. сушествует точка $\left(r_{1}, g_{1}\right) \in \Omega_{2}^{\prime \prime}$, такая что $\left(r_{1}^{\prime}, g_{1}^{\prime}\right) \notin \Omega_{2}$. Очевидно, что в области $\Omega_{2}^{\prime \prime}$ имеется точка $\left(r_{2}, g_{2}\right)$, образ которой принадлежит $\Omega_{2}$. (Пусть, например, $\left(r_{2}, g_{2}\right)=\left(r, g_{1}^{-}(r)\right)$, где $r<r_{-}(\alpha)$. Тогда $r_{2}^{\prime}=$ $r_{-}(\alpha), g_{2}^{\prime}<g_{-}\left(r_{-}\right)$.) Рассмотрим образ некоторой кривой $\gamma$, принадлежащей области $\Omega_{2}^{\prime \prime}$ и имеюшей два конца в точках $\left(r_{1}, g_{1}\right)$ и $\left(r_{2}, g_{2}\right)$. Очевидно, что должна сушествовать точка $\left(r_{3}, g_{3}\right) \in \gamma$, такая что $\left(r_{3}^{\prime}, g_{3}^{\prime}\right) \in \gamma_{2}$. Из равенства

$$
\frac{g^{\prime}}{\left(r^{\prime}+n^{\alpha-1}\right)^{2}}=n^{-1} \frac{g}{(r+1)^{2}}
$$

следует, что $n^{-1}<g_{3}^{\prime} /\left(r_{3}^{\prime}+n^{\alpha-1}\right)^{2}<1$. По теореме 1 и в силу того что отображение (1) обратимо в области $G_{2},\left(r_{3}, g_{3}\right) \in \gamma_{2}$. Полученное противоречие доказывает лемму.

Лемма 2. Пусть $\alpha \geq 3 / 2$. Тогда, если $(r, g) \in \Omega_{3}^{\prime}=\Omega_{3} \backslash G_{3}$, mo $r^{(N)} \rightarrow+\infty$. Eсли жее $(r, g) \in \Omega_{4}^{\prime}=\Omega_{4} \backslash G_{3}$, mo $r^{(N)} \rightarrow-\infty$.

ДокАЗАТЕЛЬСтво. Пусть $(r, g) \in \Omega_{4}^{\prime}$, т.е. $r<-\frac{1-n^{-1}}{1-n^{-\alpha}}, g<0$. Тогда легко доказать неравенство

$$
r^{\prime}+\frac{1-n^{-1}}{1-n^{-\alpha}}<n^{\alpha-1}\left(r+\frac{1-n^{-1}}{1-n^{-\alpha}}\right) .
$$

Отсюда $r^{(N)} \rightarrow-\infty$.

Пусть теперь $(r, g) \in \Omega_{3}^{\prime}$. Докажем, что $\left(r^{\prime}, g^{\prime}\right) \in \Omega_{3}^{\prime}$. Действительно, нетрудно проверить, что образ кривой $g=g_{2}(r)$ задается как $g=g_{2}^{\prime}(r)$, где

$$
g_{2}^{\prime}(r)=\frac{n^{-1}\left(1-n^{1-\alpha}\right) r}{\left(1-n^{-\alpha}\right) r+1-n^{-1}}\left(r+n^{\alpha-1}\right)^{2}
$$

- возрастаюшая функщия при $-\frac{1-n^{-1}}{1-n^{-\alpha}}<r<0$, при этом $g_{2}^{\prime}(r)<g_{2}(r)$. Область $\Omega_{3}^{\prime}$ при преобразовании РГ отображается во множество точек нижней полуплоскости, лежаших правее кривой $g=g_{2}^{\prime}(r)$ и, следовательно, принадлежащих $\Omega_{3}^{\prime}$. 
В силу формулы (3) из доказанного следует, что если $(r, g) \in \Omega_{3}^{\prime}$, то последовательность $r^{(N)}$ возрастаюшая. Допустим, что сушествует конечньй предел $r^{(\infty)}$ последовательности $r^{(N)}$. Очевидно, что $r^{(\infty)}>-\frac{1-1 / n}{1-n^{-\alpha}}$ и $g^{(N)} \rightarrow \infty$ (в противном случае мы бы имели неподвижную точку в нижней полуплоскости, что при $\alpha \geq 3 / 2$ неверно). Переходя в тождестве

$$
r^{(N+1)}=n^{\alpha-1}\left(\frac{\left(r^{(N)}+1\right)^{2}-g}{\left(r^{(N)}+1\right)^{2}-g / n}\left(r^{(N)}+1\right)-1\right)
$$

к пределу при $N \rightarrow \infty$, получим соотношение

$$
r^{(\infty)}=n^{\alpha-1}\left(n\left(r^{(\infty)}+1\right)-1\right)
$$

которое не может быть выполнено при $r^{(\infty)}>-\frac{1-1 / n}{1-n^{-\alpha}}$. Лемма доказана.

Лемма 3. Пусть $\alpha>1 ; g^{(N)} \neq 0$ nри всех $N ; \quad \beta^{(N)}<d<1$ nри всех $N>N_{0}$ и $r^{(N)} \rightarrow+\infty$ или $r^{(N)} \rightarrow-\infty$. Тогда существуют константы $c_{1}\left(r^{(0)}, g^{(0)}\right) \neq 0$ и $c_{2}\left(r^{(0)}, g^{(0)}\right) \neq 0$, maкuе что

$$
\lim _{N \rightarrow \infty} \frac{r^{(N)}}{n^{N(\alpha-1)}}=c_{1}, \quad \lim _{N \rightarrow \infty} \frac{g^{(N)}}{n^{N(2 \alpha-3)}}=c_{2}
$$

ДокАЗАТЕЛьство. Докажем сначала, что

$$
\beta^{(N)}=o\left(n^{-N / 2}\right) .
$$

Пусть $D=\frac{1-d}{1-d / n}$. Очевидно, что $D>0$ и $\sigma^{(N)}>D$ при всех $N>N_{0}$. Имеем

$$
\frac{\beta^{(N)}}{\beta^{(N+1)}}=n\left(1-\frac{n^{\alpha-1}-1}{n^{\alpha-1} \sigma^{(N)}\left(r^{(N)}+1\right)}\right)^{2} \rightarrow n,
$$

откуда следует (8).

Из (8) вытекает, что

$$
1-\sigma^{(N)}=o\left(n^{-N / 2}\right)
$$

Так как $\sigma^{(N)} \neq 0$ для всех $N$ (по условию леммы $g^{(N+1)} \neq 0$ ), то из последнего равенства вытекает сушествование предела $L$ бесконечного произведения $\prod_{N=0}^{\infty} \sigma^{(N)}$. Из формулы (6) следует, что $c_{2}=L^{2} g^{(0)}$.

Нам осталось доказать существование константы $c_{1}$. Рассмотрим последовательности

$$
a_{ \pm}^{(N)}=\frac{r^{(N)} \pm 1}{n^{N(\alpha-1)}} .
$$

Из соотношения (5) вытекает неравенство

$$
a_{+}^{(N+1)}<\sigma^{(N)} a_{+}^{(N)} .
$$

Кроме того, из (9) следует, что

$$
\sigma^{(N)}>\frac{n^{\alpha-1}+1}{2 n^{\alpha-1}}
$$


при всех $N \geq N_{0}^{\prime}$. Легко проверить, что неравенство (11) равносильно неравенству

$$
a_{-}^{(N+1)}>\sigma^{(N)} a_{-}^{(N)} .
$$

Докажем, что из неравенств (10), (12) следует утверждение леммы. Рассмотрим случай $r^{(N)} \rightarrow \infty, g^{(0)}>0$ (все остальные случаи рассматриваются аналогично). Тогда $0<\sigma^{(N)}<1$ при всех $N>N_{0}$. Из неравенства (10) вытекает сушествование предела (возможно, равного нулю) последовательности $a_{+}^{(N)}$. Однако последовательности $a_{ \pm}^{(N)}$ имеют один и тот же предел $c_{1}$. Из неравенства $(12)$ и того, что $L \neq 0$, следует, что $c_{1}>0$. Лемма доказана.

ДОКАЗАТЕЛЬСТво теОРемЫ 2. Рассмотрим сначала случай $\alpha>3 / 2,(r, g) \in \Omega_{1}$. Область $\Omega_{1}$ в этом случае представляется в виде объединения следующих непересекаюшихся частей:

$$
\begin{aligned}
& H_{1}=\left\{(r, g): \quad 0<r \leq r_{+}(\alpha), \quad 0<g<h_{+}(r ; \alpha)\right\}, \\
& H_{2}=\left\{(r, g): \quad r_{+}(\alpha)<r, \quad g_{2}(r)<g<h_{+}(r ; \alpha)\right\}, \\
& H_{3}=\left\{(r, g): \quad r_{+}(\alpha)<r, \quad g_{3}^{+}(r) \leq g \leq g_{2}(r)\right\}, \\
& H_{4}=\left\{(r, g): \quad r_{+}(\alpha)<r, \quad g_{5}^{+}(r) \leq g<g_{3}^{+}(r)\right\}, \\
& H_{5}=\left\{(r, g): \quad r_{+}(\alpha)<r, \quad 0 \leq g<g_{5}^{+}(r)\right\},
\end{aligned}
$$

где

$$
g_{5}^{ \pm}(r)=n^{-1} \frac{n^{\alpha-1 / 2} \mp n}{n^{\alpha-1 / 2} \mp 1}\left(r+n^{\alpha-1}\right)^{2} .
$$

Заметим, что парабола $g_{5}^{+}(r)$ проходит через точку $\left(r^{+}, g^{+}\right)$, а парабола $g_{5}^{-}(r)$ - через "минусовую" неподвижную точку. Из соотношения (7) и леммы 1 следует, что

$$
\begin{aligned}
& \left(r^{\prime}, g^{\prime}\right) \in H_{1} \cup H_{5}, \quad \text { если } \quad(r, g) \in H_{1} \cup H_{4} \cup H_{5}, \\
& \left(r^{\prime}, g^{\prime}\right) \in H_{2} \cup H_{3} \cup H_{4}, \quad \text { если }(r, g) \in H_{2} \cup H_{3} .
\end{aligned}
$$

Легко проверить, что $g_{+}(r)<g_{2}(r)<g_{3}^{+}(r)$ при $0<r<r_{+}(\alpha)$. Из формул (3), (4) следует, что если $(r, g) \in H_{1} \cup H_{4} \cup H_{5}$, то $r^{\prime}>r$ и $g^{\prime}>g$; если $(r, g) \in H_{2}$, то $r^{\prime}<r$ и $g^{\prime}<g$; если $(r, g) \in H_{3}$, то $r^{\prime} \geq r$ и $g^{\prime} \leq g$. Таким образом, дополняя (13), (14), можно написать, что

$$
\begin{aligned}
& \left(r^{\prime}, g^{\prime}\right) \in H_{3} \cup H_{4}, \quad \text { если }(r, g) \in H_{3}, \\
& \left(r^{\prime}, g^{\prime}\right) \in H_{5}, \text { если }(r, g) \in H_{4} \cup H_{5} .
\end{aligned}
$$

Если для всех $N \geq N_{0}$ выполнено $\left(r^{(N)}, g^{(N)}\right) \in H_{i}$, где $i$ фиксировано и равно 1,2 или 3 , то в силу монотонности последовательностей $\left(r^{(N)}\right.$ и $\left.g^{(N)}\right)$ сушествует конечный предел $\lim _{N \rightarrow \infty}\left(r^{(N)}, g^{(N)}\right)=\left(r^{(\infty)}, g^{(\infty)}\right)$. Этот предел должен являться неподвижной точкой отображения $(1)$, поэтому $\left(r^{(\infty)}, g^{(\infty)}\right)=\left(r_{+}, g_{+}\right)$. Однако этот факт противоречит единственности устойчивой инвариантной кривой в окрестности “плюсовой” неподвижной точки. 
Поэтому из (13)-(16) следует, что если $(r, g) \in \Omega_{1}$, то $\left(r^{(N)}, g^{(N)}\right) \in H_{5}$ для всех $N>N_{0}$. При этом последовательность $r^{(N)}$ при $N>N_{0}$ возрастающая и неограниченная (в противном случае мы бы имели неподвижную точку в области $\Omega_{1}$, что неверно). Применяя лемму 3 , получаем утверждение теоремы.

Случай $3 / 2 \geq \alpha>1,(r, g) \in \Omega_{1}$ рассматривается аналогично.

Пусть теперь $\alpha>1,(r, g) \in \Omega_{2}$. Область $\Omega_{2}$ разбивается на следующие непересекаюшиеся части:

$$
\begin{aligned}
& H_{1}^{\prime}=\left\{(r, g): \quad r_{-}(\alpha)<r<-1, \quad(r, g) \in \Omega_{2} \cap G_{2}\right\}, \\
& H_{2}^{\prime}=\left\{(r, g): \quad r_{-}(\alpha)<r<-1, \quad g_{3}^{-}(r)<g \leq g_{-}(r)\right\}, \\
& H_{3}^{\prime}=\left\{(r, g): \quad-\infty<r<r_{-}(\alpha), \quad g_{2}(r)<g \leq g_{1}^{-}(r)\right\}, \\
& H_{4}^{\prime}=\left\{(r, g): \quad-\infty<r<-1, \quad \max \left(0, g_{3}^{+}(r)\right) \leq g \leq \min \left(g_{2}(r), g_{3}^{-}(r)\right)\right\}, \\
& H_{5}^{\prime}=\left\{(r, g): \quad-\infty<r<-1, \quad 0 \leq g<g_{3}^{+}(r)\right\} .
\end{aligned}
$$

Заметим, что $H_{5}^{\prime}=\varnothing$, если $\alpha \leq 3 / 2$.

Из теоремы 1 и леммы 1 следует, что для любой точки $(r, g) \in H_{1}^{\prime}$ сушествует такое $N$, что $\left(r^{(N)}, g^{(N)}\right) \in H_{2}^{\prime} \cup H_{3}^{\prime} \cup H_{4}^{\prime} \cup H_{5}^{\prime}$.

Легко видеть, что область $H_{2}^{\prime}$ при преобразовании РГ перейдет в область

$$
\left\{(r, g): \quad-n^{\alpha-1}<r<r_{-}(\alpha), \quad g_{5}^{-}(r)<g \leq g_{-}^{\prime}(r)\right\},
$$

где

$$
g_{-}^{\prime}(r)=\frac{r\left(r+n^{\alpha-1}\right)}{r+n^{\alpha-1}(1-\sqrt{n})}
$$

- образ кривой $g_{-}(r)$. Можно показать, что $g_{-}^{\prime}(r)<g_{1}^{-}(r)$ при $r<r_{-}(\alpha)$. Следовательно, если $(r, g) \in H_{2}^{\prime}$, то $\left(r^{\prime}, g^{\prime}\right) \in H_{3}^{\prime} \cup H_{4}^{\prime} \cup H_{5}^{\prime}$.

Из формул (2)-(4) следует, что если $(r, g) \in H_{3}^{\prime}$, то $r^{\prime}>r, g^{\prime}<g$ и $\beta^{\prime} \leq \beta$; если $(r, g) \in H_{4}^{\prime}$, то $r^{\prime} \leq r, g^{\prime} \leq g$ и $\beta^{\prime}<\beta$; если $(r, g) \in H_{5}^{\prime}$, то $r^{\prime}<r, g^{\prime} \geq g$ и $\beta^{\prime} \leq \beta$.

Отсюда если $(r, g) \in H_{3}^{\prime}$, то $\left(r^{\prime}, g^{\prime}\right) \in H_{3}^{\prime} \cup H_{4}^{\prime} \cup H_{5}^{\prime}$; если $(r, g) \in H_{4}^{\prime}$, то $\left(r^{\prime}, g^{\prime}\right) \in H_{4}^{\prime} \cup$ $H_{5}^{\prime}$; если $(r, g) \in H_{5}^{\prime}$, то $\left(r^{\prime}, g^{\prime}\right) \in H_{5}^{\prime}$.

Рассуждая так же, как и в случае области $\Omega_{1}$, можно показать, что для всех точек $(r, g) \in \Omega_{2}$, для которых $g^{(N)} \neq 0$, выполнены условия леммы 3. Отсюда вытекает справедливость доказываемой теоремы.

Утверждение теоремы 2 для областей $\Omega_{3}$ и $\Omega_{4}$ легко следует из теоремы 1 и лемм 1, 2, 3 .

Заметим, что из доказанной теоремы следует, что при выполнении ее условий

$$
\beta^{(N)}=O\left(n^{-N}\right) .
$$

Поскольку все грассманово-значные плотности вида

$$
f\left(\psi^{*}\right)=a+b\left(\bar{\psi}_{1} \psi_{1}+\bar{\psi}_{2} \psi_{2}\right)+c\left(\bar{\psi}_{1} \psi_{1} \bar{\psi}_{2} \psi_{2}\right)
$$

отличаюшиеся друг от друга константным множителем, задают одно и то же состояние, мы можем рассматривать набор констант $(a, b, c)$ как точку двумерного проективного пространства. При этом $\delta$-функции соответствует набор $(0,0,1)$, а плотности $f\left(\psi^{*} ; r, g\right)$ - набор

$$
\left(1,-r, r^{2}-g\right) \equiv\left(r^{-2},-r^{-1}, 1-\frac{g}{r^{2}}\right) .
$$

Если точка $(r, g)$ удовлетворяет условиям теоремы 1 , то из (17) и того, что $\left|r^{(N)}\right| \rightarrow \infty$, следует, что области $\Omega_{1}, \Omega_{2}, \Omega_{3}, \Omega_{4}$ при соответствуюших значениях $\alpha$ лежат в зоне притяжения неподвижной точки $(0,0,1)$.

2 Теоретическая и математическая физика, т. 107, № 2, 1996 г. 


\section{3. ТЕРМОДИНАМИЧЕСКИЙ ПРЕДЕЛ В ФЕРМИОННОЙ ИЕРАРХИЧЕСКОЙ МОДЕЛИ}

Напомним, что термодинамический предел фермионной модели существует, если существует предел всех корреляционных функций при $N \rightarrow \infty$.

Преж де всего опишем множество $S_{N}$ нулей статсуммы $Z_{N}(r, g)$, лежащих в областях $\Omega_{1}, \Omega_{2}, \Omega_{3}, \Omega_{4}$. Из доказанной в [1] рекуррентной формулы

$$
\begin{aligned}
Z_{N}(r, g) & =\text { const } C(r, g)^{n^{N-1}} Z_{N-1}\left(r^{\prime}, g^{\prime}\right), \\
C(r, g) & \left.=(r+1)^{2}-g\right)^{n-2}\left((r+1)^{2}-g / n\right), \\
Z_{0}(r, g) & =\left(\left(r+\frac{1-n^{\alpha-2}}{1-n^{-1}}\right)^{2}-g\right),
\end{aligned}
$$

следует, что все точки из $S_{N}$ должны удовлетворять соотношениям

$$
g^{(N)}=\left(r^{(N)}+\frac{1-n^{\alpha-2}}{1-n^{-1}}\right)^{2}
$$

или

$$
g^{(i)}=\left(r^{(i)}+1\right)^{2} \text { для некоторого } i \leq N-1
$$

при $n>2$. Заметим, что парабола $g=n(r+1)^{2}$ не лежит в рассматриваемых областях. Из (17) следует, что для любой точки $(r, g)$ из этих областей соотношение (18) не может быть выполнено при достаточно больших $N$. Пусть

$$
\begin{gathered}
S=\bigcup_{N \geq 0}\left\{(r, g) \in \Omega_{2}: g^{(N)}=\left(r^{(N)}+1\right)^{2}\right\}, \text { если } n>2 ; \\
S=\varnothing, \text { если } n=2 .
\end{gathered}
$$

Tеорема 3. Пусть $2>\alpha>1 u(r, g) \in \Omega_{1} \cup \Omega_{2} \backslash S$ uлu $2>\alpha \geq 3 / 2 u(r, g) \in$ $\Omega_{3} \cup \Omega_{4}$. Тогда модели, описьваемые гамильтонианом $H_{0, N}(\bar{\psi}, \psi, \alpha)+H_{N}(\bar{\psi}, \psi ; r, g)$, имеют термодинамический предел.

ДокаЗАТЕльство. Достаточно доказать существование предела одноточечных корреляционных функций (см., например, [2])

$$
\begin{aligned}
& u_{N}^{1}(r, g)=\rho_{N}(r, g)\left(\psi_{1}(i) \bar{\psi}_{1}(i)+\psi_{2}(i) \bar{\psi}_{2}(i)\right), \\
& u_{N}^{2}(r, g)=\rho_{N}(r, g)\left(-\psi_{1}(i) \bar{\psi}_{1}(i) \psi_{2}(i) \bar{\psi}_{2}(i)\right), \quad i \in \Lambda_{N},
\end{aligned}
$$

при $N \rightarrow \infty$. По лемме 1 работы [1] вектор $u_{N}(r, g)$ удовлетворяет рекуррентному соотношению

$$
\begin{gathered}
u_{N}(r, g)=A(r, g) u_{N-1}\left(r^{\prime}, g^{\prime}\right)+s(r, g), \\
A(r, g)=\frac{1}{n}\left(\begin{array}{cc}
\frac{\partial r^{\prime}}{\partial r} & \frac{\partial g^{\prime}}{\partial r} \\
\frac{\partial r^{\prime}}{\partial g} & \frac{\partial g^{\prime}}{\partial g}
\end{array}\right), \quad s(r, g)=\frac{1}{n C(r, g)}\left(\begin{array}{c}
\frac{\partial C(r, g)}{\partial r} \\
\frac{\partial C(r, g)}{\partial g}
\end{array}\right),
\end{gathered}
$$


с начальным условием

$$
u_{0}(r, g)=\frac{1}{Z_{0}(r, g)}\left(\frac{\frac{\partial Z_{0}(r, g)}{\partial r}}{\frac{\partial Z_{0}(r, g)}{\partial g}}\right)
$$

Таким образом,

$$
u_{N}(r, g)=\sum_{i=1}^{N-1} h_{i}(r, g)+v_{N}(r, g)
$$

где

$$
\begin{aligned}
h_{i}(r, g) & =A(r, g) \times \cdots \times A\left(r^{(i-1)}, g^{(i-1)}\right) s\left(r^{(i)}, g^{(i)}\right), \\
v_{N}(r, g) & =A(r, g) \times \cdots \times A\left(r^{(N-1)}, g^{(N-1)}\right) u_{0}\left(r^{(N)}, g^{(N)}\right) .
\end{aligned}
$$

Используя (17) и то, что $\left|r^{(N)}\right| \rightarrow \infty$, легко показать, что

$$
\lim _{N \rightarrow \infty} s\left(r^{(N)}, g^{(N)}\right)=0, \quad \lim _{N \rightarrow \infty} u_{0}\left(r^{(N)}, g^{(N)}\right)=0
$$

для всех точек из рассматриваемой области. Кроме того, можно проверить, что для любых точек $(r, g)$

$$
\begin{gathered}
\operatorname{det} A(r, g)=n^{3 \alpha-6} \sigma^{3}, \\
\operatorname{Sp} A(r, g)=\frac{2}{n-1}\left(n^{\alpha-2}\left(\sigma^{2}-\sigma \frac{n+3}{2}+n\right)-n^{2 \alpha-4} \sigma\left(\sigma^{2}-\sigma \frac{3 n+1}{2}+n\right)\right),
\end{gathered}
$$

так что при $\sigma=1$ собственные числа матрищы $A(r, g)$ суть $n^{\alpha-2}$ и $n^{2 \alpha-4}$. Так как $\sigma_{N} \rightarrow 1$, то начиная с некоторого номера $N$ спектральная норма матрицы $A\left(r^{(N)}, g^{(N)}\right)$ будет меньше некоторого числа $t<1$. Отсюда нормы векторов $h_{i}(r, g)$ и $v_{N}(r, g)$ удовлетворяют неравенствам

$$
\left\|h_{i}(r, g)\right\|<\mathrm{const} t^{i}, \quad\left\|v_{N}(r, g)\right\|<\operatorname{const} t^{N} .
$$

Сходимость ряда (19) следует из оценок (20). Теорема доказана.

Пусть $u_{\infty}(r, g) \equiv \lim _{N \rightarrow \infty} u_{N}(r, g)$ суть корреляционные функции поля на всей решетке. Из того факта, что $s\left(r^{(N)}, g^{(N)}\right) \rightarrow 0$, и того, что $\left\|A\left(r^{(N)}, g^{(N)}\right)\right\|<t<1$ для всех достаточно больших $N$, следует, что

$$
\lim _{m \rightarrow \infty} u_{\infty}\left(r^{(m)}, g^{(m)}\right)=0 .
$$

Это и означает, что автомодельный предел фермионного поля на всей решетке с потенциалом $f\left(\psi^{*} ; r, g\right)$ является вырожденным полем с потенциалом $\delta\left(\psi^{*}\right)$ для всех $(r, g)$, удовлетворяющих условиям последней теоремы.

Автомодельные пределы для точек $(r, g)$, лежаших на инвариантных кривых $\gamma_{1}$ и $\gamma_{2}$ при $\alpha>3 / 2$ задаются потенциалами, отвечающими "плюсовой" и "минусовой" неподвижным точкам, соответственно. Динамика преобразования РГ в области между $\gamma_{1}$ и $\gamma_{2}$ будет исследована в следующей работе. Результаты, описанные в этой статье, будут использованы при классификации критических явлений и вычислении критических индексов в данной модели.

Работа выполнена при поддержке Российского фонда фундаментальных исследований (код проекта 95-01-00270) и Международного научного фонда (код гранта RHA300). 


\section{Список литературы}

[1] Lerner E.Yu., Missarov M.D. // J. Stat. Phys. 1994. V. 76. № 3/4. P. 805-817.

[2] Лернер Э.Ю., Миссаров М.Д. // ТМФ. 1994. Т. 101. С. 282-293.

[3] Bleher P.M., Sinai Ya.G. // Commun. Math. Phys. 1973. V. 33. P. $23-42$.

[4] Collet P., Eckmann J.-P. A renormalization group analysis of the hierarchical model in statistical mechanics. Lect. Notes in Phys. V. 74. Berlin-Heidelberg-New York: Springer Verlag, 1978.

[5] Синай Я.Г. Теория фазовых переходов. М.: Наука, 1980.

[6] Gavedzki K., Kupiainen A. // Commun. Math. Phys. 1985. V. 1. P. 102.

[7] Ydman J., Magnen J., Rivasseu V., Seneor R. // Commun. Math. Phys. 1986. V. 103. P. 67.

[8] Dorlas T.C. // Commun. Math. Phys. 1991. V. 136. P. 169.

[9] Березин Ф.А. Метод вторичного квантования. М.: Наука, 1986.

[10] Марсден Дж., Мак-Кракен М. Бифуркация рождения цикла и ее приложения. М.: Мир, 1980.

Казанский государственный

Поступила в редакцию университет 16.VIII.1995 г.

\section{E.Yu. Lerner, M.D. Missarov \\ GLOBAL FLOW OF RENORMALIZATION GROUP AND THERMODYNAMIC LIMIT FOR THE HIERARCHICAL FERMIONIC MODEL}

The renormalization group transformation for the hierarchical fermionic model is given as a rational map in the plane of the coupling constants. We investigate the global flow of this transformation. The existence of the thermodynamic limit in the domain under investigation is proved. The automodel limit of the constructed fields is described. 